\title{
Carnets
}

Revue électronique d'études françaises de l'APEF

Deuxième série - 21 | 2021

Arts du vin

\section{Boire ou ne pas boire, telle est la question}

\section{Maria do Rosário Girão}

\section{(2) OpenEdition}

\section{Journals}

Édition électronique

URL : https://journals.openedition.org/carnets/12835

DOI : $10.4000 /$ carnets. 12835

ISSN : 1646-7698

Éditeur

APEF

\section{Référence électronique}

Maria do Rosário Girão, « Boire ou ne pas boire, telle est la question », Carnets [En ligne], Deuxième série - 21 | 2021, mis en ligne le 31 mai 2021, consulté le 01 juin 2021. URL : http://

journals.openedition.org/carnets/12835; DOI : https://doi.org/10.4000/carnets.12835

Ce document a été généré automatiquement le 1 juin 2021.

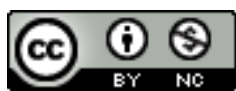

Carnets est mis à disposition selon les termes de la licence Creative Commons - Atribution - Pas d'utilisation commerciale 4.0 International. 


\title{
Boire ou ne pas boire, telle est la question
}

\author{
Maria do Rosário Girão
}

1 À l'instar de l'amour et de la guerre, on chante le vin depuis l'Antiquité classique (le "pramnio » des poèmes homériques) jusqu'à nos jours. Cette suprématie vinicole est évidente dans l'œuvre de Caton (apud Pereira, 2015 : 12), selon qui le vin guérit la sciatique et combat l'indigestion; dans l'œuvre de Virgile, qui consacre une partie considérable du livre II des Géorgiques (II, 2-8) au choix du sol propice à la culture du cep de vigne et au temps idéal pour le planter $(1997: 86)$; dans les Odes d'Horace $(1,7)$, qui conseille Planco à mettre fin aux vicissitudes de la vie en compagnie du vin $(2008: 61)$; dans les épigrammes en distiques de Martial, ayant comme sujet les vins de bonne et de mauvaise qualité : en se rapportant aux vins de Sorrente, de Tarragone et de Spolète, il rehausse l'excellence du légendaire «Falerno del Massico » et la médiocrité de celui de Ravenne (III, 56) et du Vatican (VI, 92); dans le Satyricon [Satyrica] de Pétrone, où l'amphitryon Trimalcion, pour qui « La vie est le vin» $(2000: 306)$, surtout le « Falerno Opimiano ", prône l'abolition de l'eau au profit de la consommation du vin de la Campanie; dans le poème no 14 de la collection Carmina Burana (Codex Buranus), qui évoque la taverne comme un espace équivoque de réjouissance et de perdition, où personne ne craint la mort et tout le monde rend hommage à Bacchus, car l'eau était insalubre, au contraire du vin qui n'était jamais infecté. D'ailleurs, du point de vue de Bertrand Russel (1961 : 38), il ne faut pas s'étonner de ce culte bachique, car les Grecs avaient développé le goût du primitif et d'une vie plus instinctive et passionnée que celle sanctionnée par la morale courante.

2 En parcourant les siècles, on constate que le vin, au Moyen Âge, était la boisson par excellence, fort connue depuis l'époque romaine, remplacée, en dernière instance, par la bière, quand son prix devenait exorbitant (Laurioux, 1992: 65-69). Sous la plume rabelaisienne (et "Rire est le propre de l'Homme»), sous l'égide du grotesque, synonyme d'imagination carnavalesque, et sous le signe de la boisson nait ${ }^{1}$, à l'époque de la Renaissance, Gargantua, buveur pacifique qui conseille la consommation d'un «trait » afin de soulager les reins; d'ailleurs, pour boire, « il n'avait ni fin ni règles » 
(Rabelais, 1995: 68). Dans un ton plus sérieux, l'astrologue-médecin-humaniste Nostradamus a rédigé une ordonnance, destinée à l'un de ses clients allemands, qui constitue un véritable hymne à la vie et au vin : «Faites en sorte de vous bien porter, abandonnez-vous tout entier à la gaieté (...), à l'ardeur, fuyez les différends et les querelles, (...) Servez-vous du bon vin, du vieux (...)» (apud Colonna, 2002 : 36). L'acmé de cette joie de vivre est atteinte au moment où Dom Pérignon, moine et maitre des caves de l'Abbaye de Hautvillers, préside au développement du champagne, introduit dans la cour de Louis XIV, à Versailles, par l'entremise des pratiques confessionnelles. Considéré démodé par Louis XVI, il connaît son apogée lors du sacre de Napoléon Bonaparte, ami intime de Jean-Rémy Moët, et son plein essor au XIX siècle, vingt-cinq ans après la défaite de Waterloo (New Holland Publishers, 1999). Toutefois, l'alcool, le vin en particulier, a un statut ambivalent. À ce propos, Nietzsche soutient le principe de la dualité et de la réconciliation de Dionysos et d'Apollon, de la réalité et de l'illusion, du vin et du soleil, de l'ivresse et de la raison, de la démesure et de l'harmonie, débouchant sur une nouvelle perception esthétique et artistique: "Nous nous rapprochons maintenant du véritable objectif de notre recherche, qui est de connaître et de pénétrer le génie et l'œuvre d'art dionyso-apolliniens, ou tout au moins de pressentir la nature de cette mystérieuse unité. » (1993 : 47-48). Piquette létale ou nectar divin, dénommé, renommé ou anonyme, aphrodisiaque ou fade, rouge ou blanc, ayant ou n'ayant pas le label "Appellation d'origine contrôlée », le vin connote la sagesse (en ce qui concerne sa production), la civilisation (par rapport à sa qualité) et la convivialité (relativement aux rituels familiaux et aux étiquettes sociales).

3 Gaston Bachelard, dans une perspective philosophique, souligne que le vin est suc de soleil et de terre, son état de base étant non pas l'humide, mais le sec : «Mieux que tout autre végétal, la vigne trouve l'accord des mercures de la terre (...). Elle travaille tout le long de l'année en suivant la marche du soleil à travers tous les signes zodiacaux. » (1979 : 325). Cela étant, le vin se situe aux antipodes de l'eau, ce qui est vrai du point de vue mythique : « Ainsi la nature a pris soin - bonne mère ! - d'interdire par la force des treilles l'union des liquides contraires, l'union de l'eau et du vin, l'union de la mare et du coteau. » (idem : 326 ). Tout en ratifiant mythiquement, et non pas sociologiquement, l'affirmation bachelardienne, le sémiologue Roland Barthes envisage le vin comme une "boisson-totem", supportant "une mythologie variée qui ne s'embarrasse pas des contradictions» (1970: 69). Du côté symbolique, le vin, (breuvage de vie ou d'immortalité) associé au sang vital, par sa couleur, et au sacrifice mortel, par son effusion, devient le symbole de la connaissance et de l'initiation, à cause de l'ivresse qu'il déclenche (Chevalier \& Gheerbrant, 1982 : 1016). Celle-ci, liée tout d'abord aux moissons et aux récoltes, est tantôt physique, tantôt spirituelle, la première s'avérant un moyen d'accès à la deuxième, voire un transport des facultés mentales. La première, l'ivresse physique, s'identifie à un "empoisonnement momentané ", l'alcoolâtre finissant par " changer la nature de son sang », et par vicier ou perdre, en conséquence, ses facultés générales. C'est la thèse de Balzac qui, dans son Traité des excitants modernes, envisage l'ivresse, sous quelque forme qu'elle se manifeste, comme étant la « négation du mouvement social» $(1981$ : 326) et la définit comme un plaisir - malgré ou à cause de ses excès ? - qui obnubile la vie réelle, qui éteint la «connaissance des peines et des chagrins » (idem : 314 ) et qui libère l'être humain du «fardeau de la pensée », ce qui justifie l'usage qu'en font les hommes de génie et les gens du peuple. 
4 En tant que népenthès ${ }^{2}$, assimilé parfois et souvent à l'opium et au haschisch, le statut $\mathrm{du}$ vin est métaphorique et oxymorique, détenant quelques fonctions, valeurs et dimensions qui, loin d'être isolées, fonctionnant en compartiments étanches, s'interpénètrent dans la plupart des cas.

\section{Une fonction sociale}

5 «On a bu et chanté le vin pendant bien des siècles " - écrit Brillat-Savarin dans Physiologie du goût (1848: 114). Pour ce physiologiste, le vin - «la plus aimable des boissons " (ibidem), due à Noé qui a planté la vigne, ou, alors, à Bacchus, figure de création mythique/divine - date de l'enfance du monde, du temps des origines ou du temps primordial, pour paraphraser Mircea Eliade. Après avoir affirmé qu'il n'y a pas plaisir de table si le vin est mauvais, en mettant sur un pied d'égalité la nourriture et la boisson, quasi (con-) fondues, après avoir vanté le vin de Sauternes et déclaré que les vins de Grèce, de Sicile et d'Italie ont fait les délices des Romains, il se tourne vers le Portugal vinophile : "Après le clairet vint le porto, après le porto le madère, auquel nous nous tînmes longtemps » (idem : 332). Faisant suite à ce contexte viticole, il ne résiste pas à la joyeuse tentation de raconter un épisode exquis, à l'image du vin auquel il se rapporte : «Un buveur était à table. Et au dessert on lui offrit du raisin. 'Je vous remercie', dit-il en repoussant l'assiette, 'je n'ai pas la coutume de prendre mon vin en pilules'" (idem: 386). De même, les fins thérapeutiques remontent à la surface: si Brillat-Savarin affirme, d'une manière pragmatique, que les vins blancs, légers et acidulés sont indiqués pour le traitement de l'obésité, Flaubert (pour qui le vin est un " sujet de conversation entre hommes ") est d'avis que le meilleur vin est le bordeaux, «puisque les médecins l'ordonnent». Et il ajoute: «Plus il est mauvais, plus il est naturel.» (1961: 150). Cette fonction en convoque, nécessairement, une autre, qui lui est intrinsèque, étant donné que le vin instaure un décor, fonde une socialité, séduit les ouvriers, les bourgeois et les aristocrates qu'il discrimine en castes. En feuilletant la flaubertienne Éducation Sentimentale (1869) ${ }^{3}$, on est invité à côtoyer la société à table moyennant la description de trois dîners. Le premier a lieu chez les Arnoux : « Il (...) eut à choisir entre dix espèces de moutarde. Il mangea du daspachio, du cari, du gingembre, des merles de Corse, des lasagnes romaines; il but des vins extraordinaires, du lip-fraoli et du tokay.» (1983: 64-65). Le deuxième se déroule chez Rosanette : «Les vins circulaient, les plats se succédaient. (...) On lançait de loin une orange, un bouchon ; (...) » (idem : 144). Le troisième est servi à la Maison d'Or :

Un surtout de vermeil, chargé de fleurs et de fruits, occupait le milieu de la table, couverte de plats d'argent, suivant la vieille mode française ; (...) des cruches de vin rosat frappé de glace se dressaient de distance en distance ; cinq verres de hauteur différente étaient alignés devant chaque assiette (...) (idem : 241).

Faisant foi à l'apophtegme, que nous adaptons, "Dis-moi ce que tu bois, je te dirai ce que tu es ", l'éclectisme œnologique de M. Arnoux, qui se "piquait de bien recevoir ", traduit par le mélange de vins du Rhin et de la Hongrie, renvoie par métonymie à son caractère bohème, passionné d'un exotisme hétéroclite et de vocables ou noms surprenants. En revanche, chez la cocotte Rosanette, l'indéfinition ou l'indétermination redoutables des marques du vin, associées au lancement d'un bouchon, peignent soit le comportement des convives soit une société qui aurait pu partager « l'oie de la fête de Gervaise ou la ripaille de Nana " (Cogny, 1975: 157). À la Maison d'Or, l'apparat inhérent à la hauteur variable ou à la place symétrique des verres cristallise le faux- 
semblant d'un soi-disant luxe et constitue une critique à l'idée et à l'image de l'illusion de vivre dans une société éteinte.

\section{Une dimension psychologique et libératrice}

7 D'après Thomas De Quincey ${ }^{4}$, plusieurs ouvriers de Manchester avaient l'habitude de prendre de l'opium, car leurs maigres revenus ne leur permettaient pas d'acheter de la bière ou de l'alcool. Lui-même, grâce à ses Confessions, est devenu l'opiomane anglais le plus connu', détenteur d'une sensibilité 'audio-visuelle', qui, d'après Michèle Stäuble, est enrichie par la sensibilité olfactive et tactile de Baudelaire, son traducteur et adaptateur. Dans ce contexte, l'essayiste eudémoniste (qui aspire au bonheur, le sien et celui des autres, et abomine la misère) procède à l'étude comparative entre les effets du vin et de l'opium, cherchant à dédouaner celui-ci des calomnies dont il a été victime, mais aussi à définir leurs différences.

8 Tout d'abord, le plaisir causé par le vin, un plaisir aigu que la flamme métaphorise, s'intensifie dans une ligne ascendante, jusqu'au moment où il commence à diminuer, tandis que le plaisir dû à l'opium, un plaisir chronique métaphorisé par le feu constant, reste égal à lui-même tout au long de huit ou dix heures environ. Ensuite, le vin vole à l'homme le gouvernement de soi-même, perturbe ses facultés mentales, voile son jugement, exacerbe ses sentiments (l'amitié, l'amour et la haine), fortifie son intelligence, rend sa conscience plus aiguë, volatilise ses énergies intellectuelles, fait appel à l'animalité de son être et enlève son masque, en livrant son intériorité, son vrai caractère ; par opposition au vin, l'opium harmonise les facultés humaines, actives ou passives, en leur prêtant l'équilibre et la sérénité, apaise n'importe quelle agitation momentanée, concentre la tendance à la dispersion et impose la suprématie de la spiritualité.

9 Cette idée de nocivité inhérente aux excitants, l'alcool au premier chef, est également partagée par Balzac qui s'empresse d'énumérer quelques effets néfastes du vin, à savoir, l'hébétement du cerveau, au lieu de son activation, l'obscurcissement des papilles, la saturation des conduits et la perte du goût, ainsi que l'incapacité du buveur à distinguer la finesse des liquides servis. Donc, écrit Balzac en s'adressant et en avertissant son lecteur, «inscrivez cet axiome dans votre mémoire : L'ivresse est un empoisonnement momentané » (1994: 23). Et pourtant, faisant suite à son statut ambivalent, à la fois nectar et poison, le vin a une fonction libératrice, mise en scène par Flaubert dans deux contes bachiques de jeunesse, intitulés respectivement « Ivre et Mort » (1838) et «Les funérailles du Docteur Mathurin » (1839). Dans le premier, deux amis se lancent un défi pour déterminer qui boira le plus jusqu'à atteindre une agonie ivre et désespérée. Dans le deuxième, le Docteur Mathurin, imprégné d'une philosophie rabelaisienne, épicurienne et sceptique, décide, aux approches de la mort, de franchir joyeusement le seuil de la vie, en compagnie de deux disciples fidèles et de plusieurs bouteilles de vin. Loin des rumeurs de la ville et des bruits du monde, le protagoniste attend, dans une somnolence bénéfique, la mort, afin de lui donner, en guise de dernier orgasme, « des baisers pleins de vin, un cœur plein de la vie et qui n'en veut plus. - Un cœur ivre et qui ne bat pas. ». «Que la mort vienne, qu'elle vienne (...) » - s'exclame-t-il (Flaubert, 1991 : 340). Et quand, enfin, elle survient, son cadavre ivre, transporté par ses amis, traverse la ville au sein de l'indignation et du scandale de la foule - «aucun roi n'eut jamais tant de monde à ses funérailles » (idem : 349) -, est salué par « un hymne au 
vin, à la nature, au bonheur, à la mort » et se voit arrosé par le vin : « (...) la terre le but vite et alla porter jusqu'à Mathurin les souvenirs des dernières saveurs de son existence et réchauffer sa tête couchée sur la terre!» (idem: 353). L'explicit de ce conte, correspondant, en dernière analyse, à l'arrêt du convoi funèbre peu orthodoxe et à la fin du rituel hétérodoxe qu'il sous-entend, illustre, à tous les égards, l'orgie alcoolique et tabagique ante-mortem et post-mortem: "On ne vit plus que les restes de deux bouteilles - (...) elles rappelaient des joies et montraient un vide. » (ibidem).

Il convient, dans cette conjoncture, de mettre en relief le pèlerinage initiatique de $\mathrm{M}$. Jean Folantin, protagoniste de À vau-l'eau de Huysmans, à travers une vie et une ville un «Paris » haussmannien qui « devient un Chicago sinistre » (1882:16) -, en quête de marchands de vin, de vins moins âpres, exécrables ou misérables (idem : 11 et 20), de vins qui ne fleurent pas la benzine (idem: 36 ) et de quelques apéritifs des cafés, moins abjects, susceptibles d'aiguiser son appétit, d'atténuer son trou d'ennui et sa nausée existentielle : «(...) les absinthes puant le cuivre; les vermouths; la vidange des vins blancs aigris ; les madères (...)» (idem : 12). Dégoûté par l'univers environnant et vaincu par le vide qui le menace, il prend la sage décision de s'empiffrer de «la charcuterie chez soi », «boire de l'eau. » (idem : 21) et se « laisser aller à vau-l'eau » (idem : 40), en faisant sienne la maxime pessimiste de Schopenhauer, pour qui la vie de l'homme oscille comme un pendule entre la douleur et l'ennui. En fait, le vin volatilise la personnalité de l'homme, éparpille l'empire de l'être humain sur lui-même, mais catalyse des énergies et condense des peurs.

\section{Une valeur morale}

11 Du point de vue de Roland Barthes, le vin, qui est une fonction, «fonde une morale collective, à l'intérieur de quoi tout est racheté : les excès, les malheurs, les crimes sont sans doute possibles avec le vin, mais nullement la méchanceté, la perfidie ou la laideur ; le mal qu'il peut engendrer est d'ordre fatal » $(1970: 72)^{6}$.

12 Il convient de souligner, dans cet ordre d'idées et en réitérant ce que nous avons déjà affirmé, que le vin ruine en même temps qu'il libère: on glorifie ses bienfaits (la dissipation de la mélancolie, la conquête du plaisir), on atténue ses méfaits (les malheurs et les crimes qu'il engendre). Baudelaire semble, à ce propos, exemplaire, moyennant le dialogue / conflit que, à travers son œuvre, l'on est en mesure d'établir avec Balzac et Flaubert, démontrant leurs appréciations différentes à l'égard de BrillatSavarin. Du côté balzacien, la critique de l'auteur de Le Lys dans la vallée, assez faible, à l'auteur de Physiologie du goût (1755-1826) se penche uniquement sur un défaut d'insistance susceptible de combler des omissions.

Brillat-Savarin, qui l'un des premiers a remarqué l'influence de ce qui entre dans la bouche sur les destinées humaines, aurait pu insister sur l'utilité d'élever la statistique au rang qui lui est dû, en en faisant la base sur laquelle opéreraient de grands esprits. (1994: 44-45).

D'ailleurs, la dénonciation balzacienne des lacunes de Brillat-Savarin est récurrente dans le Traité des excitants modernes : il ne remarque pas « la liaison qui existe entre les produits de l'homme et les substances qui peuvent changer la condition de sa vitalité (idem : 13) ; à propos du café, en tant qu'excitant, il « est loin d'être complet » (idem : $25)$; en outre, il est inouï qu'il «ait oublié le chapitre du tabac» (idem: 35). Ces reproches, du point de vue de Raymond Mahieu, qui suit les pas de P.-G. Castex ${ }^{7}$, 
"surprendraient dans une préface ", mais « n'ont plus rien qui dérange dès lors qu'on a affaire à un texte autonome, qui ne se réfère à celui qu'il accompagne que pour mieux marquer qu'il ne lui doit rien » (1994: 87).

Du côté flaubertien, l'éloge - que fait Flaubert - de Brillat-Savarin glisse vers le degré de parenté et de proximité de son œuvre relativement à celle de Horace et de Rabelais : "Ils ne recevaient ni livres, ni journaux, ni lettres, la bibliothèque commune se composait d'Horace, de Rabelais. Ai-je besoin de dire qu'il y avait toutes les éditions de Brillat-Savarin et du Cuisinier?» (1991: 338). En fait, selon le mot de Flaubert/ Mathurin, la gastronomie et la philosophie forment « deux sciences éternelles » dans le traité savarinien intitulé Méditations de gastronomie transcendante (1825).

Quant à Baudelaire, l'attaque contre Brillat-Savarin, personnelle, culturelle et littéraire, s'endurcit, l'ironie féroce s'aiguisant et traduisant son mépris à l'égard tantôt de la renommée de l'homme - "très-célèbre", "grand sot", "oracle du goût", « infaillible »-, tantôt de son soi-disant chef-d'œuvre, qu'il ne faut absolument pas lire :

Ah! Chers amis, ne lisez pas Brillat-Savarin. (...) Si une nouvelle édition de ce faux chef-d'œuvre ose affronter le bon sens de l'humanité moderne, buveurs mélancoliques, buveurs joyeux, vous tous qui cherchez dans le vin le souvenir ou l'oubli, (...) achèterez-vous un exemplaire et rendez-vous le bien pour le mal, le bienfait pour l'indifférence? (Baudelaire, 1979 : 304).

La pomme de discorde ne réside que dans un renseignement " précieux », « digestif » et très explicatif » (par antiphrase), voire "banal », « indigeste » (c'est-à-dire susceptible de ne pas être assimilé, dans le sens métaphorique) et d'une incomplétude et décontextualisation contraignantes :

Un homme très célèbre (...) a osé (...) écrire ce qui suit à l'article VIN : 'Le patriarche Noé passe pour être l'inventeur du vin, c'est une liqueur qui se fait avec le fruit de la vigne.' Et après? Après rien : c'est tout. Vous aurez beau feuilleter le volume, le retourner dans tous les sens, le lire à rebours, à l'envers, de droite à gauche et de gauche à droite, vous ne trouverez pas autre chose sur le vin (...) (ibidem : 304).

Voilà, peut-être, deux faces de la même monnaie : d'une part, les défauts de la position de Brillat-Savarin, qui aurait dû, selon Baudelaire, approfondir, encadrer et développer la référence biblique; d'autre part, les excès de Baudelaire, qui fait preuve d'une agressivité contondante par rapport à la superficialité qui caractérise quelques passages de l'essai du magistrat Brillat-Savarin.

Dans son essai intitulé " Du vin et du haschisch ", daté d'une période où le poète était emporté par des considérations sociales - sous la Monarchie de Juillet, la misère, « montrant comme des travailleurs s'aggrave et la bourgeoisie s'enrichit - débouchant sur une tendance socialiste et humanitaire ${ }^{8}$, il avoue qu'il est accusé d'innocenter l'ivrognerie (et il ne dément pas ce réquisitoire), parce que, devant les bienfaits, il n'a pas le courage de compter les griefs. A vrai dire, et dans sa perspective, le vin rajeunit les gens fatigués de la lutte quotidienne, devient l'espoir bienheureux des dimanches, roule, comme un nouveau Pactole, un or intellectuel à travers l'humanité, compose des chants et des poèmes afin d'apaiser la foule souffrante et ennoblit les choses les plus viles, devenant un rituel puisqu'il « récidive tous les jours ». Ritualisé et objet de culte, il répète jour après jour ses bienfaits, ce qui explique l'acharnement des moralistes contre lui ou, mieux, des pseudo-moralistes pharisiens (idem: 305). Semblable et assimilable à l'homme, il ne pourrait jamais disparaitre de son horizon, d'après le poète de Les Fleurs du Mal, sous peine de creuser « dans la santé et dans l'intellect de la planète un vide, une absence, une défectuosité beaucoup plus affreuse que tous les excès et les 
déviations dont on rend le vin responsable » (idem : 306). Et Baudelaire va encore plus loin, lorsqu'il écrit, d'une façon catégorique, que les abstèmes, ceux qui ne boivent que de l'eau, sont des imbéciles ou des hypocrites, gardant dans leur for intérieur un secret à cacher à leurs semblables. Parfois, c'est le lait - véritable anti-vin, du point de vue sociologique, pour Barthes ${ }^{9}$ - qui donne lieu à ce rôle négatif attribué, par Baudelaire, à l'eau. Attiré, un jour, par un "objet monstrueux » dans une Exposition de peinture voire un tableau s'avérant l'antithèse de l'art absolu, au contraire de "Cuisine » de Drölling $^{10}$ - et, par conséquent, avide de renseignements sur le caractère moral de son auteur, Baudelaire constate que « le monstre se levait régulièrement avant le jour, qu'il avait ruiné sa femme de ménage, et qu'il ne buvait que du lait ! En vérité, l'homme et le vin sont indissociables, ils ne font qu'Un, tantôt en dialogue amoureux, tantôt en conflit meurtrissant. Il y a, bien sûr, des ivrognes méchants, mais leur méchanceté est naturelle, car, sous les effets du vin, l'homme méchant devient exécrable, comme l'homme bon devient excellent (idem : 308).

Et voilà que surgissent, aux antipodes des «profondes joies du vin » et de la grandeur des «spectacles du vin illuminés par le soleil intérieur " (idem : 304-305), ses effets à craindre, ses "voluptés foudroyantes » et ses «enchantements énervants ». Tout en nous penchant sur les poèmes "Le vin de l'assassin » et "Le vin des amants », il est légitime de constater qu'ils constituent un diptyque thématique: le premier étale le côté dangereux du vin, en incitant au meurtre et en visant à la conquête de la liberté. En d'autres termes, la liberté ne peut être acquise qu'après la disparition de l'amour :

Ma femme est morte, je suis libre ! Je puis donc boire tout mon soûl. / (...) - Me voilà libre et solitaire! / Je serai ce soir ivre mort ; /Alors sans peur et sans remords, /Je me coucherai sur la terre; / (...) Écraser ma tête coupable/ Ou me couper par le milieu, /Je m'en moque comme de Dieu, /Du Diable ou de la Sainte Table ! // (1979: 109-110).

Quant au deuxième, il offre au lecteur une 'navigation' aérienne, «à cheval sur le vin ", en compagnie d'une femme-sœur et à destination du « mirage lointain » et du " paradis de [mes] rêves». Cela étant, "Le vin des amants", poème mystique, exalte la quintessence du délire d'amour redoublé par le vin, tandis que « Le vin de l'assassin », à l'instar du projet de la pièce théâtrale "L'Ivrogne", des Contes immoraux de Pétrus Borel et de la nouvelle de Edgar Allan Poe intitulée Le démon de la perversité1 ${ }^{11}$, fait « du vin un linceul », en glorifiant sa démesure satanique.

\section{Une mission artistique, poétique et alchimique}

21 Il est indéniable que le vin ouvre la voie aux transpositions et correspondances artistiques, voire à l'Art, la musique en l'occurrence, en nourrissant son imaginaire. C'est le cas de Hoffmann, dans ses Fantaisies à la manière de Callot ou, plutôt, dans ses Kreisleriana, attribuées par lui à son alter ego, le maître de chapelle Johannès Kreisler, et source d'inspiration pour Robert Schumann. Quelques conseils donnés par l'écrivaincompositeur allemand à tous les musiciens consciencieux ne doivent pas être passés sous silence (apud Baudelaire, 1979 : 304) : en fait, Hoffmann leur recommande, en effet, le vin de Champagne pour composer un opéra-comique, boisson où ils peuvent trouver la joie que le genre réclame; la musique religieuse, par son amertume enivrante, demande du vin du Rhin ou du Jurançon ; à la musique héroïque, à cause de la fougue sérieuse et de l'entraînement patriotique, fait défaut le vin de Bourgogne. Outre cette fraternité entre la composition musicale et les qualités musicales du vin, Hoffmann 
dresse un baromètre psychologique susceptible de représenter les phénomènes atmosphériques de son esprit: l'esprit légèrement ironique, l'esprit de solitude et l'aspiration à sortir de son moi. À son tour, Balzac, qui considère le vin le plus ancien des excitants - «à tout seigneur, tout honneur »-, raconte un épisode curieux, basé sur la correspondance entre le vin et la musique, dont il été le protagoniste au Théâtre Italien en 1822 :

Ce monsieur sent le vin', dit à voix basse une dame dont le chapeau effleurait souvent ma joue, et que, à mon insu, ma joue allait effleurer. J'avoue que je fus piqué.

- 'Non, madame, répondis-je, je sens la musique' (1994 : 20).

Plusieurs années plus tard, c'est le tour de Huysmans, créateur de l'esthète décadent Des Esseintes, de métaphoriser "des barils à liqueurs » en "orgue intérieur », en établissant une relation de similitude et assimilation entre l'alcool et l'instrument de musique, ou, mieux, en faisant correspondre chaque liqueur, en termes de goût, au son d'un instrument, jouant de la sorte des symphonies intérieures à partir de chaque goutte. Cela étant, le curaçao sec équivaut à la clarinette, le kummel au hautbois, la menthe et l'anisette à la flûte et "pour compléter l'orchestre, le kirsch sonne furieusement de la trompette. » (1978: 99-100). Cette correspondance entre la musique et le vin, à la fois catalyseur de sensations inédites et condensateur de nouveaux drames (Rincé, 1984 : 50), n'est possible que grâce au pouvoir transfigurateur du verbe, à la « sorcellerie évocatoire », à la poésie et au sujet poétique.

Dans Répertoire I, Michel Butor fait une affirmation, à propos du haschisch et de l'opium, que l'on peut appliquer au vin : «De même que seule la poésie pouvait donner quelque durée à ce qui se dévoilait sous l'exemple du haschich, de même seule la poésie peut donner à l'individu quelque pouvoir sur cette matière onirique que l'opium déchaîne " $(1979$ : 119). Dans un registre moins sérieux, Butor, grand connaisseur de La Comédie Humaine, amuse le lecteur avec ses drolatiques Scènes de la vie excitante, où l'on peut trouver, bien sûr, quelques références à Brillat-Savarin : «Un accusateur public prépare un bol de café qui met en mouvement le sang, comme l'a fort bien observé Brillat-Savarin, pour Marthe Michu en l'interrogeant sur un caveau caché sous les ruines de l'ancien monastère de Nodesme..$^{12} »$ (Butor, 1994 : 73). Dans un autre passage, l'écrivain, avec humour, s'empresse de mentionner un oubli ${ }^{13}$ de l'auteur de Physiologie $d u$ goût: «(...) Auguste présente à la duchesse de Langeais, qui le méprise, un petit cigare de tabac dont Brillat-Savarin a oublié le chapitre dans son ouvrage. » (idem : 67). Ce reproche, concernant l'incomplétude, est souligné, par Butor, à la fin de cet essai comique: "Maxime de Trailles (...) ayant développé (...) une passion pour le chocolat, autre excitant moderne auquel l'auteur du Traité, s'il le mentionne, n'a sans doute pas accordé toute l'attention qu'il mérite, meurt dans un effroyable de pourriture (...)» (idem : 78). Et que dire de la poésie du vin ? Ou de l'ivresse poétique?

Dans l'œuvre baudelairienne, le vin a, en fait, une vertu régénératrice, surgissant comme le sujet (au lieu du buveur) de la prosopopée «L'âme du vin » et s'identifiant au poète moyennant des images évocatrices. En effet, il allume les yeux de la femme, il réconforte son fils, il se transmue en huile pour l'athlète et, de pair avec l'être humain, il crée la poésie : "En toi je tomberai, végétale ambroisie, / grain précieux jeté par l'éternel Semeur, /Pour que de notre amour naisse la poésie/Qui jaillira vers Dieu comme une rare fleur !/ » $(1979: 107)$. A son tour, dans le sonnet « Le vin du solitaire », le sujet poétique affirme la suprématie du vin sur la femme galante, la libertine Adeline, le sac d'écus «entre les doigts d'un joueur» et la musique «énervante et 
câline ", c'est-à-dire, sa supériorité sur la femme, l'argent et la musique : «Tout cela ne vaut pas, ô bouteille profonde, /Les baumes pénétrants que ta panse féconde /Garde au cœur altéré du poète pieux (...) » (idem : 110). D'une part, il s'associe et se transforme en ce poète solitaire et pieux, chantant les bienfaits du vin; d'autre part, il risque de pécher par orgueil, en rivalisant avec les Dieux, ce qui devient la conséquence la plus grave de l'ivresse : « Tu lui verses l'espoir, la jeunesse et la vie, / - Et l'orgueil, ce trésor de toute gueuserie, /Qui nous rend triomphants et semblables aux Dieux!» (idem:110). En fin de compte, le vin, la poésie et le sujet poétique ont le pouvoir philosophique et alchimique de transmuter la boue en or et de créer ex nihilo. Deux répondants allégoriques résument le statut poétique et démiurgique du vin: le chiffonnier et Napoléon Bonaparte. Le chiffonnier amasse les débris et ramasse les ordures d'une journée de travail, voire tout ce que la ville a rejeté ou perdu ou, encore, qui est considéré comme abject : « Il compulse les archives de la débauche, le capharnaüm des rebuts.» (idem: 305). Ensuite, il en fait le tri, il sélectionne, il ordonne ces matières premières pour les transformer, pour en faire des objets utiles ou jouissants. Finalement, il s'en va, "butant sur les pavés, comme les jeunes poètes qui passent toutes leurs journées à errer et à chercher des rimes." (ibidem). À l'image du chiffonnier, le poète part à la recherche de matières primitives, par-ci, par-là, les décompose et les collectionne, avant de choisir, par une «solennelle magie " (idem : 109), celles qui conviennent soit à la transformation alchimique soit à la création poétique. De même, à l'instar de Napoléon, figure christique et mythique, oscillant entre l'ascension et la chute, il rêve de glorieux projets, inachevés, peut-être, de faits admirables, incomplets, sans doute, et de victoires imaginaires, pouvant ou ne pouvant pas devenir réelles. Derrière l'homme se profile le vin, excitant et stimulant, permettant d'augmenter la personnalité du pascalien "roseau pensant » et de créer, par une opération mystique, une troisième personne : ainsi, l'homme et le vin, le dieu animal et le dieu végétal, engendrent-ils un Saint-Esprit, qui est l'homme supérieur, le créateur ou l'artiste par excellence.

D'après Bachelard, les trois règnes de la vie alchimique sont le règne minéral (l'or étant le roi des métaux), le règne végétal (monde intermédiaire qui a la vigne comme reine, car elle attire l'or solaire) et le règne animal (dont le liquide noble est le sang). Or, même si le vin n'entre pas dans ces catégories, il est tout de même de nature alchimique. Cela étant, « l'esprit du vin » est une image dont la substance, matérielle et immatérielle, présente les caractéristiques de l'opération alchimique. Extrait de la vie des substances, «l'esprit du vin », détenteur d'une sagesse semblable à la quintessence de la materia prima des alchimistes, est une métaphore de la perfection de soi que l'or alchimique symbolise : "(...) le vin blanc est or potable. Le vin rouge est un sang." (1979: 329). En outre, vivre est un art, l'art de bien vieillir, parallèlement au vin, qui maîtrise, également, l'art de savoir mûrir en vieillissant : "Le vin est vraiment un universel qui sait se rendre singulier, s'il trouve, toutefois, un philosophe qui sache le boire. » (idem : 332 ).

Dans un siècle où la gastronomie et l'alcool jouent un rôle capital et où «l'emploi que les romanciers font du discours gastronomique semble osciller entre l'identification et la mise en question » (Becker, 2017 : 178), le vin apparaît au centre de plusieurs débats herméneutiques, offrant une pluralité louable de points de vue. Aux antipodes de l'eau et associée au sang vital, cette " boisson-totem », qui déclenche une ivresse physique et spirituelle, une illusion fallacieuse d'éternité que le retour au réel mine constamment, a une fonction sociale, une dimension psychologique et libératrice, une valeur morale et 
une mission philosophique ${ }^{14}$, artistique, poétique et alchimique. Il suffit de rappeler les théories de Brillat-Savarin, de Balzac, de Flaubert et de Baudelaire autour non seulement de Physiologie du goût, mais aussi du statut ambivalent des excitants modernes. Pour Balzac, le vin « a dans son alcool un principe nuisible ; selon Flaubert, il devient nectar et poison en simultané ; quant à Baudelaire, «Il faut être toujours ivre. (...) De vin, de poésie ou de vertu, à votre guise.» (1979:173).

En fait, le vin devient un tremplin pour l'imaginaire qui, selon Wunenburger, permet de « nous détacher de l'immédiat, du réel présent et perçu » (2003:69), de « créer pour le plaisir une autre image du monde » (idem : 75) et de « rendre disponibles des techniques de pensée symbolique et analogique » (idem: 79). Par sa vertu cathartique, par son usage métaphorique et oxymorique, par sa valeur régénératrice, par sa fonction poiétique et prégnance symbolique, le vin devient une vraie méthode de création.

\section{BIBLIOGRAPHIE}

APOLLINAIRE, Guillaume (1965). Euvres Poétiques. Paris : Gallimard, coll. « Bibliothèque de la Pléiade ». BACHELARD, Gaston (1979). La terre et les rêveries du repos. Paris : Librairie José Corti.

BALZAC, Honoré (1994). Traité des excitants modernes. Textes de Pierre Alechinsky et de Michel Butor. Arles : Actes Sud. Voir, aussi, BALZAC, Honoré (1981). La Comédie Humaine (Études analytiques). Paris : Gallimard, coll. « Bibliothèque de la Pléiade », vol. XII.

BARBIER, Auguste (1859). Iambes et Poèmes. Paris : Ed. Dentu.

BARTHES, Roland (1970). Mythologies. Paris : Seuil.

BAUDELAIRE, Charles (1976). Un mangeur d'opium. Avec le texte parallèle des Confessions of an English Opium-Eater et des Suspiria de profundis de Thomas De Quincey. Édition critique et commentée par Michèle Stäuble-Lipman Wulf (1976). À la Baconnière-Neuchâtel, coll. « Langages ».

BAUDELAIRE, Charles (1979). CEuvres Complètes. Paris : Seuil.

BECHER, Karin (2017). Gastronomie et littérature en France au XIXe siècle. Orléans : Éditions Paradigme. BRILLAT-SAVARIN, Jean-Anthelme (1848). Physiologie du goût. Paris : Gabriel de Gonet, Éditeur.

BUTOR, Michel (1960). Répertoire I. Paris : Les Éditions de Minuit.

BUTOR, Michel (1994). Scènes de la vie excitante. Traité des excitants modernes. Arles : Actes Sud, pp. 67-78.

CASTEX, P.-G. (1979). « Balzac et Brillat-Savarin. Sur une préface à la Physiologie du goût ». L'Année Balzacienne, pp. 7-14.

Champagne. New Holland Publishers (1999). Traduction de Ana Maria Chaves. Porto : Asa Editora. CHEVALIER, Jean \& GHEERBRANT, Alain (1982). Dictionnaire des symboles. Paris : Robert Laffont, coll. «Bouquins». 
COGNY, Pierre (1975). L'Éducation Sentimentale de Flaubert. Le monde en creux. Paris : Librairie Larousse, coll. « thèmes et textes ».

ColonNA, Michele (2002). « Itinéraire d'un prophète ». L'Histoire, nº 271, pp. 32-39.

DE QUINCEY, Thomas (1989). Confissões de um opiómano inglês. Lisboa : Contexto.

DE QUINCEY, Thomas (2008). Del asesinato considerado como una de las Bellas Artes y otros ensayos literarios. Traducción de José Rafael Hernández Arias. Madrid : Valdemar.

FLAUBERT, Gustave (1961). Dictionnaire des idées reçues. Paris : Aubier, Éditions Montaigne.

FLAUBERT, Gustave (1983). L'Éducation Sentimentale. Paris : Gallimard, coll. « Folio ».

FLAUBERT, Gustave (1991). Mémoires d'un fou. Novembre et autres textes de jeunesse. Paris : GF Flammarion.

HORÁCIO (2008). Odes. Traduction de Pedro Braga Falcão. Lisboa : Livros Cotovia.

HUYSMANS, Charles-Marie-Georges (1991). À rebours. Paris : GF - Flammarion.

HUYSMANS, Charles-Marie-Georges [Joris-Karl] (1882). À vau-l'eau. Livres \& Ebooks.

INOUE, Teruo (1977). Une poétique de l'ivresse chez Charles Baudelaire. Tokyo : Librairie-Éditions.

LAURIOUX, Bruno (1992). A Idade Média à mesa. Mem Martins : Forum da História.

MAHIEU, Raymond (1994). Lecture de Raymond Mahieu. Traité des excitants modernes. Arles : Actes Sud, pp. 79-98.

MARCIAL (1997). Epigramas. Traducción y notas de Juan Fernández Valverde y Antonio Ramirez de Verger. Madrid : Editorial Gredos, S. A., vol. I/II.

NIETZSCHE, Friedrich (1993). «La naissance de la tragédie ou hellénisme », CEuvres. Traduit de l'allemand par Jean Marnold et Jacques Morland. Paris : Robert Laffont.

PEREIRA, Virgínia Soares (2015). «O vinho na tradição literária romana ». Voar mais alto, n 7, pp. 12-14.

PETRÓNIO (2000). O Satirícon. Tradução de Jorge de Sampaio. Mem Martins : Publicações EuropaAmérica.

PICCHI, Fernando (1974). La droga in due esperienze parallele Baudelaire et De Quincey. Rocca San Casciano : Casa Editrice Licinio Cappelli S.p.A.

RABELAIS, François (1995). Gargantua. Paris : Flammarion.

RINCÉ, Dominique (1984). Baudelaire et la modernité poétique. Paris : Presses Universitaires de France.

VERGílio (1997). Geórgicas. Tradução de Agostinho da Silva. Lisboa : Temas e Debates.

WUNENBURGER, Jean-Jacques (2003). L'Imaginaire. Paris : Presses Universitaires de France.

\section{NOTES}

1. Nouveau-né, il s'écrie à haute voix : «À boire! À boire ! à boire » (Rabelais, 1995 : 35). Bon vivant, il aime la "purée septembrale» ... En ce qui concerne Pantagruel, il faut revisiter le "Cinquième livre», qui raconte la fin du voyage du héros en quête de l'oracle de la Dive Bouteille. 
2. Dans l'antiquité grecque, népenthès était un breuvage qui avait la propriété, selon Homère, de dissiper le chagrin.

3. Cet article est un hommage à Flaubert et à Baudelaire, étant donné qu'on a célébré l'année dernière le centième-quarantième anniversaire de la mort de Flaubert, et que l'on fête cette année le bicentenaire de Flaubert et Baudelaire (nés en 1821).

4. L'activité littéraire de l'écrivain anglais Thomas De Quincey ne s'est pas réduite à l'étude approfondie de la consommation et des effets de l'opium. Il ne faut pas oublier ses Essais littéraires, parmi lesquels On murder considered as one of the Fine Arts (1827), dans lequel il prône la suprématie esthétique du plaisir contemplatif sur le plaisir moral, ainsi que la non-coïncidence entre le beau et le bon, moyennant le dialogue avec la Poétique d'Aristote, Laokoon de Lessing et Philosophical Inquiry into the Sublime and Beautiful de Edmund Burke.

5. Voir, à ce propos, le portrait que Guillaume Apollinaire fait de De Quincey dans son poème intitulé « Cors de chasse » : «(...) Et Thomas de Quincey buvant/L'opium poison doux et chaste/A sa pauvre Anne allait rêvant/Passons passons puisque tout passe/Je me retournerai souvent/Les souvenirs sont cors de chasse/Dont meurt le bruit parmi le vent. » (1965: 148).

6. Roland Barthes ajoute que le « vin échappe donc à la pénalisation, c'est un mal de théâtre, non un mal de tempérament. » (ibidem).

7. Voir, à ce propos, Castex, P.-G., « Balzac et Brillat-Savarin. Sur une préface à la Physiologie du goût » (1979: 7-14). Selon Mahieu (1994:87), « on a longtemps cru que le texte de Balzac était la préface, commandée et payée anticipativement, de cette nouvelle édition [Physiologie du goût de Brillat-Savarin] ». Or, Castex rétablit la vérité, «montrant comment l'essai s'était substitué, en tant que complément à l'œuvre de Brillat-Savarin, à une préface jamais achevée (...) ».

8. Il convient de souligner, dans ce contexte, que Baudelaire est proche de Banville et de Barbier en ce qui concerne l'ivresse du vin. D'une part, le point de vue baudelairien est plus moraliste que celui de Théodore de Banville dans le poème «La chanson du vin »; d'autre part, le poète de Paris met l'accent sur la vertu transformatrice du vin/poésie, différemment de Auguste Barbier qui le chante dans un 'cadre' misérable : « Sombre génie, ô dieu de la misère ! / Fils du genièvre et frère de la bière, / Bacchus du Nord, obscur empoisonneur, /Ecoute, ô Gin, un hymne en ton honneur.» (1859: 203).

9. «Bachelard avait sans doute raison de donner l'eau comme le contraire du vin: (...) aujourd'hui, ce l'est moins ; des circonstances économiques ou historiques ont dévolu ce rôle au lait. » (1970 : 71).

10. Peintre d'origine alsacienne, Martin Drölling a acquis douze cœurs (des membres de la famille royale) lors de la Révolution Française (et après celle-ci). Selon la légende, les peintres avaient l'habitude d'en extraire une substance précieuse, la " mummie ", qui, mêlée à l'huile, donnait un glacis incomparable aux tableaux. Ce tableau (peinture de genre) a été peint en 1815.

11. Dans The Imp of the Perverse, nouvelle traduite par Baudelaire, Poe ne définit pas la perversité dans un sens moral, mais plutôt comme étant synonyme d'esprit de contradiction.

12. Voir, à ce propos, la reprise (moyennant l'inversion), par Butor, de l'affirmation de Balzac: «Comme l'a fort bien observé Brillat-Savarin, le café met en mouvement le sang, en fait jaillir les esprits moteurs (...)» (idem : 25)

13. Cet oubli est, également, emprunté à Balzac, qui le dénonce dans la partie IV (DU TABAC) de son essai (idem : 35 ).

14. Plusieurs philosophes et écrivains ont écrit des maximes, des proverbes et des phrases qui, désormais célèbres, constituent un vrai florilège vinophile : «In vino veritas_» (Pline) ; « L'ivresse ne déclenche pas les vices, mais les met en relief » (Sénèque); «Dans l'eau nous reflétons notre image, dans le vin nous lisons l'image d'autrui » [proverbe] ; «Dieu a créé l'eau, l'homme a fait le vin » (Victor Hugo »; «Le vin est la poésie embouteillée » (Robert Louis Stevenson) ; «Je boirai du lait le jour où les vaches mangeront du raisin » (Jean Gabin). 


\section{RÉSUMÉS}

«N'avons-nous pas tous nos gourmandises?» - s'interroge Alphonse Karr dans son «Introduction » à l'œuvre de Brillat-Savarin intitulée Physiologie du goût. En fait, on chante le vin depuis Homère et l'Antiquité jusqu'aux temps modernes : il suffit de survoler le $\mathrm{xx}^{\mathrm{e}}$ siècle et de s'arrêter sur les Confessions de De Quincey, les 'paradis' d'Edgar Allan Poe, la correspondance hoffmannienne entre le vin et la musique, le traité balzacien des excitants modernes et sur la poétique flaubertienne / baudelairienne de l'ivresse par le vin, physique ou libératrice, spirituelle ou mystique. En tant que népenthès, assimilé à l'opium et au haschisch, l'usage du vin est métaphorique et oxymorique du point de vue social, dans une perspective morale et en ce qui concerne sa mission philosophique et alchimique. "Boisson-totem », selon Barthes, sa fonction esthétique débouche sur la multiplication de l'individualité et sur la création d'une troisième personne, l'homme supérieur ou l'artiste par excellence.

"Don't we all have our delicacies? " Alphonse Karr wonders in his «Introduction " to The Physiology of Taste by Brillat-Savarin. Indeed, we've lauded wine from Homer and the Classical Antiquity to modern times: all we have to do is take a quick look at the 20th century and stop at De Quincey's Confessions, Edgar Allan Poe's "paradises ", the Hoffmannian correspondence between wine and music, the Balzacian Treatise on Modern Stimulants and the Flaubertian / Baudelairean physical or liberating, spiritual or mystical poetics of drunkenness through wine. As nepenthes, identified with opium and hashish, the use of wine is metaphorical and oxymoric from a social viewpoint, from a moral perspective, and when it comes to its philosophical and alchemical mission. A " Totem Drink », according to Barthes, its aesthetic function leads to the multiplication of individuality and the creation of a third person, the superior man or the artist par excellence.

\section{INDEX}

Mots-clés : vin, ivresse, valeur morale, alchimie, création littéraire

Keywords : wine, drunkenness, moral value, alchemy, literary creation

\section{AUTEUR}

\section{MARIA DO ROSÁRIO GIRÃO}

Un. do Minho - CEHUM -APEF

rosario[at]ilch.uminho.pt 